\title{
MATHEMATICAL MODELING OF RAINWATER RUNOFF OVER CATCHMENT SURFACE AND MASS TRANSFER OF CONTAMINANT INCOMING TO WATER STREAM FROM SOIL
}

\author{
A. Chekalin*, M. Khramchenkov, V. Konyukhov, I. Konyukhov, A. Garaeva \\ Kazan Federal University
}

Published online: 08 August 2017

\begin{abstract}
The subject of an article is the mathematical modeling of the rainwater runoff along the surface catchment taking account the transport of pollution which permeates into the water flow from a porous media of soil at the certain areas of this surface. The developed mathematical model consists of two types of equations: the equations for calculating of the water layer thickness over the slope surface given the precipitation and evaporation, and equation of the mass transfer of impurity coming into the surface water during its filtration in zone of incomplete saturation of soil. The model also takes into account a reverse process adsorption of impurity in soil with its low concentration or in the uncontaminated soil. Water content in the zone of incomplete saturation is determined within the approximate approach based on the model of capillary impregnation. The principal features of nonlinear differential equations are theoretically studied. In particular, it is shown that the equation for water content in this zone can become a differential equation with lagging argument.
\end{abstract}

Keywords: Mathematical Modeling, Runoff, Transport of Contaminant, Catchment, Sorption, Desorption, Incomplete Saturation

\section{INTRODUCTION}

The investigation of the interrelated mass transfer in the rainwater runoff along the catchment surface and in the porous media of soil in the basin of rivers is of central interest for

Author Correspondence, e-mail: Anatolii.Chekalin@kpfu.ru

doi: http://dx.doi.org/10.4314/jfas.v9i2s.63 
hydrology, hydrogeology, environmental geology and other allied sciences. Surface runoff has annual periodicity, however, its creation and behavior can significantly vary in depend on the intensity of precipitation, evaporation, absorption of water in the entire area of the catchment and many other factors [1-3]. These processes are further complicated by the presence of the contaminated areas with soil containing the various types of pollution which can permeate into runoff due to desorption of contaminant from the pores of soil into the water flow. There can arise also a reverse process- adsorption of impurity in soil with its low concentration or in the uncontaminated soil. Runoff is associated with the following three types of flow: the surface flow (continuous or consisting of set of streamlets), a subsurface runoff and a groundwater runoff.

Subsurface runoff has a relatively high speed in the inclined upper layers of soil, for example, at filtration of water and its motion through macro-pores and cracks. The rate of water movement along the slope beneath the catchment surface, on the one hand, is significantly less than on this surface. On the other hand, it is much more than the filtration velocity in the aquifers.

Groundwater runoff is the water flow along the saturated aquifers to the river network. This flow represents only a small part of the river hydrograph although some amount of water can come into the river channel immediately after rainfall. In various physical and geographical areas [4-6] and even within watersheds of relatively small sizes, the runoff formation may be caused by a variety of factors. Often runoff transforms from one form to another and these changes depend on the surface relief, types of atmospheric fallout, their intensity and total amount [7-14].

The behavior of runoff along the catchment surface, the riverbed and its feeders can be investigate with mathematical modeling [15-19] and solving the corresponding forecasting problems. The models suggested by authors of these works, consist of the multi-variable nonlinear differential equations, which can be numerically solved $[18,19]$. Computation of the interrelated processes during a motion of rainwater and groundwater in the river basin is characterized also by a large amount of input data, including boundaries of watershed, the topography the catchment surface given the depth of the river network, etc.

Grid data may be prepared using any standard geoinformation systems and the number of the grid nodes usually amount to hundreds of thousands and tens of millions. The problem solution becomes more complicated at presence of areas of contamination at the catchment surface and transport of contaminant incoming to water stream from soil. So the objective of this work is extension of mathematical model $[18,19]$ of runoff in case of its contaminating, 
and the theoretical analysis of the features of this problem solution which are very useful at development special numerical schemes with high computational efficiency oriented to the parallel computing on multiprocessor computers.

\section{METHODS AND EQUATIONS}

Mathematical model of the rainwater runoff along the surface catchment was developed earlier in our previous papers $[18,19]$ in framework of the concept of two-dimensional kinematic wave. To describe the mass transfer of contaminant incoming to the water stream from the porous media of soil, this model was modified and amplified with new equations. In result, new generalized system equations can be written as following:

$$
\begin{aligned}
& \frac{\partial(U+\Theta)}{\partial t}+V_{x}+V_{y}=\varepsilon_{1}, \quad V_{x}=-U^{5 / 3} V_{1, x}, \quad V_{y}=-U^{5 / 3} V_{1, y}, \\
& \frac{\partial(C U)}{\partial t}+\frac{\partial\left(C V_{x}\right)}{\partial x}+\frac{\partial\left(C V_{y}\right)}{\partial y}=\beta-C \frac{\partial \Theta}{\partial t}, \quad h(1-m) \frac{\partial C_{s}}{\partial t}=h \beta_{s}-\beta, \\
& V_{1, x}=\frac{Z_{x}^{\prime}}{n \sqrt{|\nabla Z|},} V_{1, y}=\frac{Z_{y}^{\prime}}{n \sqrt{|\nabla Z|}}, \quad|\nabla Z|=\sqrt{Z_{x}^{\prime 2}+Z_{y}^{\prime 2}}, \quad \mathrm{Z}=\mathrm{H}+\mathrm{U}, \\
& \Theta=\sqrt{a^{2} t^{2}+b t}+a t, \quad a=0.25 m g \rho r^{2} / \mu, \quad b=0.5 m^{2} \sigma r \cos \alpha / \mu, \\
& \beta=\left\{\begin{array}{ll}
0, & U=0, \\
\lambda\left(C_{s}-C\right), & U>0,
\end{array} \quad \beta_{s}= \begin{cases}0, & \partial t=0, \\
\gamma\left(C-C_{s}\right), & \partial \Theta / \partial t>0 .\end{cases} \right.
\end{aligned}
$$

Here $x$ and $y$ are spatial coordinates; $t$ is the time; $U(x, y, t)$ is the thickness of the water layer at the catchment surface; $\Theta(x, y, t)$ is water content in coil within zone of incomplete saturation («ZIS»); $m$ is the porosity of this area; $C(x, y, t)$ is the impurity concentration in water solution of the surface flow; $C_{s}(x, y, t)$ is the similar characteristic of the water solution in the soil thin boundary layer, by which water enters the soil from earth surface; $h$ is the thickness of boundary layer; $\mathbf{V}(x, y, t)$ is the velocity vector of the surface flow; $H(x, y)$ is the ground elevation above sea level; $n$ is the roughness coefficient of the catchment surface; $\varepsilon_{1}(x, y, t)$ is the function that specifies amount of precipitation and evaporation; $\rho$ is the water density; $g$ is the gravity acceleration; $r$ is the average radius of pores in zone of incomplete saturation; $\sigma$ is the surface tension coefficient; $\alpha$ is the wetting angle; $\mu$ is the viscosity of water solution; $\beta(x, y, t)$ is desorption of impurity from the surface of pores in soil by rainwater; $\beta_{s}(x, y, t)$ is the amount of the desorbed impurity in water, moving in the 
porous medium, per unit volume of soil; $\lambda$ and $\gamma$ are coefficients of desorption; $Z_{x}^{\prime}=\partial Z / \partial x ; Z_{y}^{\prime}=\partial Z / \partial y$.

System of differential equations (1) - (4) governs the mass transfer in the catchment area $D$ with boundary $\Gamma=\Gamma_{w}+\Gamma_{r}$, where $\Gamma_{w}$ is the watershed line; $\Gamma_{r}$ is intersection of this line and the river surface at its lower course, see Fig. 1. Initial and boundary conditions are:

$U(x, y, 0)=0, C(x, y, 0)=0,\left.V_{n}\right|_{\Gamma_{w}}=0, \quad C_{s}(x, y, 0)= \begin{cases}0, & (x, y) \notin D_{c}, \\ C_{s}^{0}(x, y), & (x, y) \notin D_{c} .\end{cases}$

Here $\left.V_{n}\right|_{\Gamma_{w}}$ is the normal velocity at the boundary $\Gamma_{w} ; D_{c}$ is the area of contamination source with the initial concentration $C_{s}^{0}(x, y)$.

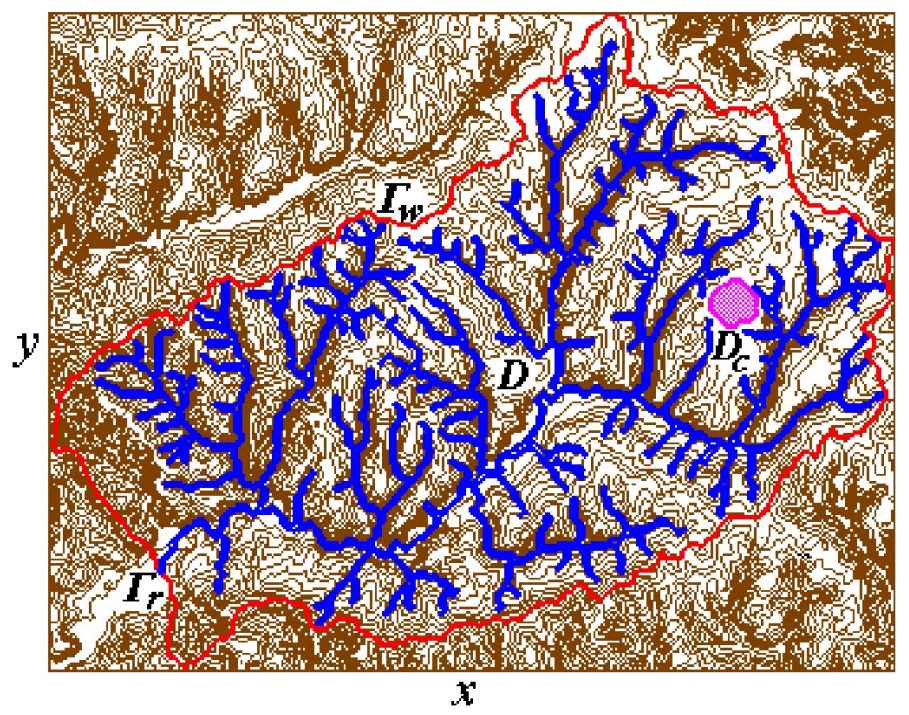

Fig.1. Topographical relief of the river basin, the bed of the river with inflows and area $D$ of the runoff formation in the presence of water pollution zone $D_{c}$ in the soil

Note 1. Parameter $h$ in the second equation (2) can be used as a verification parameter of model. It can be defined by computations in the every concrete situation. In the simplest case we can put $h=1$. Further, since absorption goes much faster than desorption so we can assume that the impurity concentration in the water solution at its moving along this thin layer is the same as in the water solution coming from the surface. In this case $\beta_{s}=\beta_{s}\left(C_{s}, C\right)$, and the desorbed impurity is instantly transported by water flow throughout the length of ZIS.

Note 2. If surface-water flow brings the impurity to the area with its lower concentration or to the area free from contaminant then the reverse process (the sorption) takes place in soil with 
coefficients $\bar{\lambda}$ and $\bar{\gamma}$, which values differ from values of $\lambda$ and $\gamma$, so that $\lambda=\left\{\begin{array}{ll}\lambda, & C_{s} \geq C, \\ \bar{\lambda}, & C_{s}<C,\end{array} \quad \gamma= \begin{cases}\gamma, & C_{s} \geq C, \\ \bar{\gamma}, & C_{s}<C .\end{cases}\right.$

Note 3. In the model (1) - (4) the impurity concentration in ZIS is not computed, however at each point $(x, y) \in D_{c}$ the total amount $\delta q(x, y, t)$ of contaminant gone with the water stream in the time interval $[0, t]$ is defined as $\delta q=\int_{0}^{t}(\beta-C \cdot \partial \Theta / \partial t) d t$. It is obvious that the value $\delta Q=\int_{D_{c}} \delta q d x d y$ is equal to amount of impurity which gone away from the area $D_{c}$.

\section{RESULTS AND DISCUSSIONS: THE PRINCIPAL FEATURES OF THE EQUATIONS SOLUTION}

Important features of the searched solution of equation (1) were studied in our work [18] in absence of impurity in the rainwater runoff. Now we generalize and reformulate obtained earlier results for the case of presence of contaminant in soil, ingress of impurity into water and its moving with the surface flow.

3.1. Since there is no inflow through the boundary $\Gamma_{w}$ of the watershed, so the thickness $U$ of the liquid layer in the area $D$ becomes greater than zero only due to the function $\varepsilon_{1}$, characterizing the intensity and the duration of rainfall. At the same time, water soaks into the soil in zone of incomplete saturation with the flow rate which is determined by the derivative $\partial \Theta / \partial t$. If precipitations have small intensity, then the pores of ZIS can completely absorb all rainwater. The absence of the surface runoff in the area $D$, i.e. equality to zero of the functions $U(x, y, t)$, reflects the inequality

$\varepsilon_{1} \leq \frac{\partial \Theta}{\partial t}=\frac{a^{2} t+b / 2}{\sqrt{a^{2} t^{2}+b t}}+a$,

which follows from equation (1) and relationship (4).

3.2. Let us consider the equation (1) at the initial time, i.e. for $t \rightarrow 0$. The surface runoff can only arise if it rains, so the limit $\left.\varepsilon_{1}\right|_{t \rightarrow 0}$ tends to the defined finite value $\varepsilon_{1}^{0}$. On the other hand, $t=0$ is the time appearance of an instant water source on the boundary of zone of incomplete saturation. The discharge flow of this source is determined by the value $\partial \theta / \partial t$. At $t \rightarrow 0$ one obtains

$\partial \Theta /\left.\partial t\right|_{t \rightarrow 0} \approx(2 a+0.5 \sqrt{b / t}) \rightarrow \infty$. 
Thus, in the initial time the condition (6) is fulfilled, and surface runoff is formed not at once but only after some time. In addition, as it follows from relation (7), the surface-water flow is missing at $\varepsilon_{1} \leq 2 a$ regardless on duration of precipitation. Note, that from the physical standpoint the parameter $2 a$ corresponds to the filtration coefficient of the soil.

3.3. The function $\theta$ (4) defines the water content in ZIS, and the derivative $\partial \theta / \partial t$ determines, on the one hand, the amount of water entering the soil through the earth surface, and on the other hand, - the velocity of the advancing front saturation in ZIS. The relationship (4) is obtained under the assumption that amount of water at the boundary of zone of incomplete saturation is enough to provide the maximum possible speed of its absorption. If the rainfall is less than they are completely absorbed by soil, and in this situation the volume of water incoming into ZIS should be determined not from the relation (4) but in a different way using the function $\varepsilon_{1}: \Theta=\int_{0}^{t} \varepsilon_{1} d \tau$.

Obviously, the velocity of the advancing front saturation will be decrease. Therefore, at $t>t_{0}$ when the intensity of rainfall will exceed the intensity of absorption and the water layer appears on the earth surface, the function $\partial \theta / \partial t$ becomes a function of the retarded argument $\tau_{d}$. In this case, it is required to define the values $t_{0}$ and $\tau_{d}$ for $\varepsilon_{1}>2 a$. Let $t_{1}=t_{0}-\tau_{d}$. At the time point $t=t_{0}$ the intensity of rainfall is equal to the absorbtion intensity:

$\frac{a^{2} t_{1}+0.5 b}{\sqrt{a^{2} t_{1}^{2}+b t_{1}}}+a=\varepsilon_{1}\left(t_{0}\right)$.

By this time, the rainfall has completely been absorbed by soil in zone of incomplete saturation forming its water content. Hence we have:

$\sqrt{a^{2} t_{1}^{2}+b t_{1}}+a t_{1}=A\left(t_{0}\right), \quad A\left(t_{0}\right)=\int_{0}^{t_{0}} \varepsilon_{1} d \tau$

Solution to the system of nonlinear equations (8) and (9) can be obtained in terms of parameters $t_{0}$ and $t_{1}$. After their transformation we write the following expressions for $t_{0}$ and $t_{1}:$

$t_{1}=\frac{b}{2 a^{2}}\left(\frac{\varepsilon_{1}\left(t_{0}\right)-a}{\sqrt{\varepsilon_{1}^{2}\left(t_{0}\right)-2 a \varepsilon_{1}\left(t_{0}\right)}}-1\right), \quad A\left(t_{0}\right)=\frac{b}{2 a}\left(-1+\frac{\varepsilon_{1}\left(t_{0}\right)}{\sqrt{\varepsilon_{1}^{2}\left(t_{0}\right)-2 a \varepsilon_{1}\left(t_{0}\right)}}\right)$. 
The values of $t_{0}$ and $t_{1}$ are calculated from the equations (10), respectively. If at $t<t_{0}$ the function $\varepsilon_{1}$ is independent of time and $\varepsilon_{1}=$ const then $a\left(t_{0}\right)=t_{0} \varepsilon_{1}$, so that the values of the lag time $t_{0}$ and the retarded argument $\tau_{d}$ are defined as:

$$
t_{0}=\frac{b}{2 a \varepsilon_{1}}\left(-1+\frac{\varepsilon_{1}}{\sqrt{\varepsilon_{1}^{2}-2 a \varepsilon_{1}}}\right), \quad \tau_{d}=t_{0}-t_{1}=\frac{b}{2 a^{2}}\left(1-\frac{a}{\varepsilon_{1}}-\sqrt{1-\frac{2 a}{\varepsilon_{1}}}\right) .
$$

Thus, the surface runoff arises only at $t=t_{0}$ and the function $\Theta$ for $t>t_{0}$ is calculated from the relationship with retarded argument:

$$
\Theta=\sqrt{a^{2}\left(t-\tau_{d}\right)^{2}+b\left(t-\tau_{d}\right)}+a\left(t-\tau_{d}\right) .
$$

3.4. The intensity of rainfall over time can become so insignificant that at an instant in time $t_{c r}$ region $D_{c r}$ with boundary $\Gamma_{c r}$ will be formed where water completely soaks into the soil. In this case, the thickness of the surface water layer $U(x, y, t)=0$ at $(x, y) \in D_{c r}, t>t_{c r}$, and the water content in the soil is defined as:

$$
\Theta(x, y, t)=\Theta\left(x, y, t_{c r}\right)+\int_{t_{c r}}^{t} \varepsilon_{1}(x, y, \tau) d \tau .
$$

At those parts $\Gamma_{c r}^{-}$of the boundary $\Gamma_{c r}$ where water flows out the region $D_{c r}$, the function $U$ is equal to zero and has no jumps. At the other parts $\Gamma_{c r}^{+}$this function can be discontinuous, and the water flow rate entering into the unsaturated zone is expressed as: $\partial \Theta / \partial t=\varepsilon_{1}+V_{n}^{+}$, where $V_{n}^{+}$is the water flow through $\Gamma_{c r}^{+}$. The area $D_{c r}$ can vanish if the intensity of precipitation will increase. In this case, as in item 3 , the values $\bar{\tau}_{d}$ and $\bar{t}_{0}$ should be calculated at the instant of time when the intensity of rainfall exceeds the intensity of absorption, and the water layer appears on the earth surface. In this case $\bar{\tau}_{d}$ and $\overline{t_{0}}$ are defined from the system that is similar to system (8), (9) and differs from these equations only in expression for $A\left(t_{0}\right)$, namely: $A\left(\overline{t_{0}}\right)=\Theta_{c r}+\int_{t_{c r}}^{\bar{t}_{0}}\left(\varepsilon_{1}+V_{n}^{+}\right) d \tau$.

3.5. At $t<t_{0}$ the surface-water flow is not formed $(U=0)$, therefore $C=0$, and in the area $D_{c}$ equation (3) allows for an analytical solution:

$C_{s}(x, y, t)=C_{s}^{0}(x, y) \cdot e^{-\gamma t /(1-m)} \approx C_{s}^{0}(x, y) \cdot(1-\gamma t /(1-m))$. 
So, the problem is formulated as follows: we need to determine the solution $U(x, y, t) \geq 0$, $\Theta(x, y, t), C(x, y, t)$ and $C_{s}(x, y, t)$ of equations (1) - (4), (10) - (13) with initial and boundary conditions (5) and estimate the water flow rate in cross-section $\Gamma_{r}$ of river at its lower course.

Discussed above a priori information on the solution behavior is very useful and will be used at development of finite-difference computational schemes to solve the system equations.

\section{SUMMARY}

The relationships and formulas to calculate of the runoff characteristics have been obtained in result of the theoretical study of the problem solution for the following typical situations.

1. The runoff may not arise in case of small intensity of precipitations when all rainwater is completely absorbed in the pores of zone of incomplete saturation.

2. Surface runoff is formed not at once with beginning of rainfall but only after some time regardless on duration of precipitation.

3. If the intensity of rainfall exceeds the intensity of absorption and the water layer appears on the earth surface, then the equation for water content in zone of incomplete saturation becomes a differential equation with lagging argument.

4. The intensity of rainfall over time can become so insignificant that at an instant in time the thickness of the surface water layer will be equal to zero within those regions of the surface catchment where water completely soaks into the soil.

5. In time period when the surface-water flow is not formed the transfer equation has an analytical solution allowing us to calculate concentration of impurity.

\section{CONCLUSIONS}

We have developed the mathematical model of runoff along the surface catchment taking account precipitation, evaporation, transport of impurity in water, its filtration in zone of incomplete saturation of soil, sorbtion and adsorption of pollutant in soil. Theoretical study of the problem solution allows us to reveal its features which will be very useful and required in developing special numerical schemes with high computational efficiency oriented to the parallel computing on multiprocessor computers. These investigations will be presented in the next our paper. 


\section{ACKNOWLEDGEMENTS}

The work is performed according to the Russian Government Program of Competitive Growth of Kazan Federal University.

\section{BIBLIOGRAPHY}

[1] S.P. Anderton, S.M. White, B. Alvera, "Micro-scale spatial variability and the timing of snow melt runoff in a high mountain catchment", J. of Hydrology, vol. 268, pp. 158-176, 2002.

[2] K.J. Beven, "Infiltration into a class of vertically non-uniform soils", Hydrological Science J., vol. 29, pp. 425-434, 1984.

[3] K.J. Beven, M.J. Kirkby, “A physically-based variable contributing area model for basin hydrology”, Hydrological Sciences Bulletin, vol. 24, №. 1, pp. 43-69, 1979.

[4] T. Dunne, T.R. Moore, C.H. Taylor, "Recognition and prediction of runoff-producing zones in humid regions", Hydrological Science Bulletin, vol. 20, pp. 305-327, 1975.

[5] P.S. Eagleson, "Dynamic Hydrology”, McGraw-Hill, New York, 1970.

[6] J.D. Hewlett, A.R. Hibbert, "Factors affecting the response of small watersheds to precipitation in humid areas", In: W.E. Sopper, H.R. Lull (eds.) Forest Hydrology, pp. 275290, Pergamon, New York, 1967.

[7] G. Jost, M. Weiler, D.R. Gluns, Y. Alila, "The influence of forest and topography on snow accumulation and melt at the watershed-scale", J. of Hydrology, vol. 347, pp. 101-115, 2007.

[8] C.H. Luce, D.G. Tarboton, K.R. Cooley, "The influence of the spatial distribution of snow on basin-averaged snowmelt”, Hydrological Processes, vol. 12, pp. 1671-1683, 1998.

[9] J.W. Pomeroy, D.M. Gray, K.R. Shook, B. Toth, R.L. Essery, A. Pietroniro, N. Hedstrom, "An evaluation of snow accumulation and ablation processes for land surface modeling", Hydrological Processes, vol. 12, pp. 2339-2367, 1998.

[10] J.W. Pomeroy, K. Dion, "Winter radiation extinction and reflection in a boreal pine canopy: measurements and modeling”, Hydrological Processes, vol. 10 (12), pp. 1591-1608, 1996.

[11] J.W. Pomeroy, R.J. Granger, A. Pietroniro, J. Elliot, B. Toth, N.R. Hedstrom, "Hydrological Pathways in the Prince Albert Model Forest", NHRI Contribution Series CS97004, Saskatoon, Saskatchewan, 1997. 
[12] J.W. Pomeroy, D.M. Gray, N.R. Hedstrom, J.R. Janowicz, "Prediction of seasonal snow accumulation in cold climate forests", Hydrological Processes, vol. 16 (18), pp. 3543-3558, 2002.

[13] C.A. Talbot, F.L. Ogden, D. Or, "Comment on Layer averaged Richards equation with lateral flow by Praveen Kumar', Adv. in Water Resources, vol. 27, pp. 1041-1042, 2004.

[14] R.D. Winkler, D.L. Spittlehouse, D.L. Golding, "Measured differences in snow accumulation and melt among clearcut, juvenile, and mature forests in southern British Columbia”. Hydrological Processes, vol. 19 (1), pp. 51-62, 2005.

[15] S.N. Antontsev, G.P. Epihov, A.A. Kashevarov, "System mathematical modeling of water exchange”, Science, Siberian Branch, Novosibirsk, 1986 (in Russian).

[16] A.I. Chebotarev, "General hydrology. (Land Water)", Gidrometeoizdat, Leningrad, 1975, (in Russian).

[17] B. Ambroise, J. Freer, K.J. Beven, "Generalization of the topmodel concepts: topographic indices of hydrological similarity", Water Resources Research, vol. 32, pp. 2135-2145, 1996.

[18] V.M. Konyukhov, A.A. Saveliev, M.G. Khramchenkov, A.N. Chekalin, N.E. Galiullina, "Mathematical and Numerical Model of the Rainwater Runoff along the Catchment Surface in the River Basin", Scientific and Technical J. Voprosy Atomnoy Nauki i Tekhniki (VANT), Seriya: Mathematical Modeling of Physical Processes, №. 3, pp. 41-54, 2013 (in Russian).

[19] V.M. Konyukhov, A.A. Saveliev, M.G. Khramchenkov, A.N. Chekalin, "Numerical Modeling of the Water Runoff along the Surface Catchment". In: XIV International Conference. Supercomputing and Mathematical Modeling. (Sarov, Russia, 2012, October, 1 5), Sarov: RFNC-VNIIEF, pp. 374-382, 2013 (in Russian).

\section{How to cite this article:}

Chekalin A, Khramchenkov M, Konyukhov V, Konyukhov I, Garaeva A. Mathematical modeling of rainwater runoff over catchment surface and mass transfer of contaminant incoming to water stream from soil. J. Fundam. Appl. Sci., 2017, 9(2S), 880-889. 